\title{
Síntesis general de arquitecturas de bloques para sistemas de almacenamiento de energía híbridos
}

\author{
Iván Sanz, Carlos Bernal, Estanis Oyarbide \\ Grupo de Electrónica de Potencia y Microelectrónica (GEPM) \\ Instituto de Investigación en Ingeniería de Aragón (I3A) \\ Universidad de Zaragoza, Mariano Esquillor s/n, 50018, Zaragoza, Spain. \\ Tel. +34-976762707, e-mail: isgorra@unizar.es
}

\begin{abstract}
En este documento se analizan desde un punto de vista general, las opciones de arquitectura en un sistema de bloques formado por dipolos y cuadripolos. El objetivo es hallar todas las configuraciones arquitectónicas posibles para un sistema de almacenamiento de energía (ESS) híbrido.
\end{abstract}

\section{Introducción}

El avance desarrollado en los últimos años en el campo del almacenamiento de energía mediante elementos electroquímicos (baterías) ha dado lugar a tecnologías de almacenamiento basadas en Litio (Ion-Litio, LiFePo4, etc.). Es conocido que estas baterías requieren de electrónica adicional para su correcto funcionamiento [1], muchas veces con capacidad de conversión, de tal manera que ya no son consideradas únicamente baterías, sino sistemas de almacenamiento completos (ESS).

El siguiente paso en esta progresiva evolución es la combinación de distintos elementos de almacenamiento dentro del mismo ESS para combatir los defectos de una única batería, como puede ser la baja densidad de potencia, o la degradación a largo plazo. Por ello, habitualmente se combinan baterías de químicas tradicionales ( $\mathrm{PbA})$ con ultracapacidades [2] con mayor densidad de potencia; o bien con baterías de químicas más nuevas (NiCd, Litio), mejores ante cambios de temperatura y degradación a largo plazo.

Estos sistemas de almacenamiento híbridos utilizan habitualmente una de las dos siguientes arquitecturas [2, 3]:

- Arquitectura de bloques en cascada, (Figura 1, a) no escalable.

- Un bus común de continua, al que conectan independientemente cada conjunto de elemento de almacenamiento y convertidor, con la desventaja del coste y complejidad por el elevado número de convertidores en funcionamiento (Figura 1, b).
Resulta por tanto de interés el análisis y síntesis de todas las configuraciones posibles de elementos de almacenamiento (dipolos) y de conversión (cuadripolos), de tal manera que se minimice el número de convertidores, mientras se mantienen las prestaciones de las arquitecturas tradicionales. Concretamente, este estudio se va a centrar en todas las arquitecturas posibles formadas por dos elementos de almacenamiento de energía, un único convertidor y el sistema a alimentar (carga).

\section{Estrategia propuesta}

A la hora de abordar el problema, se propone la creación de un conjunto de reglas que permita descartar organizadamente aquellas arquitecturas que las incumplan. De tal manera que, después de generar todas las arquitecturas posibles en una estructura de datos, se vayan purgando las configuraciones no válidas y las configuraciones redundantes, obteniendo al final el conjunto de arquitecturas realizables. Las reglas son las siguientes:

- No se permiten polos desconectados.

- No se permite cortocircuitar polos de un mismo elemento

- No se permiten baterías en serie o en paralelo con otras baterías (reducibles a una batería).

- Si intercambiando las baterías se llega una arquitectura ya conocida, esa arquitectura es redundante.

Se propone una estructura de datos matricial, en la que cada fila represente un nudo de la arquitectura, y cada columna un dispositivo (carga, batería o convertidor). Si el elemento $a_{i j}$ de la matriz es 1 , significa que el dispositivo $j$ tiene un polo conectado al nudo $i$. Si no está conectado, el elemento $a_{i j}$ será 0 .

De las reglas se derivan el número máximo y mínimo de nudos (filas) que pueden tener las arquitecturas con una carga, dos baterías y un convertidor. El número mínimo de nudos es igual al número de polos del elemento con más polos (en este caso, el convertidor, 4 polos), de tal manera que 
ninguno se cortocircuite. Por otro lado, el número de nodos máximo es igual a la suma de polos totales de todos los elementos entre 2, de tal manera que nunca haya un polo desconectado (en este caso, una carga, dos baterías y un convertidor tienen 10 polos en total, por lo tanto el número máximo de nudos es 5).

Con estas pautas, se generan todas las matrices posibles y se descartan aquellas que incumplan las normas anteriores. Un ejemplo de estas matrices se muestra en la Tabla 1, correspondiente a la arquitectura mostrada en la Figura 2, e.

Tabla 1. Ejemplo de estructura matricial de una arquitectura

\begin{tabular}{|l|c|c|c|c|}
\hline & Carga & Bat. 1 & Bat. 2 & Conv. \\
\hline Nodo 1 & 1 & 1 & 0 & 0 \\
\hline Nodo 2 & 1 & 0 & 0 & 1 \\
\hline Nodo 3 & 0 & 1 & 0 & 1 \\
\hline Nodo 4 & 0 & 0 & 1 & 1 \\
\hline Nodo 5 & 0 & 0 & 1 & 1 \\
\hline
\end{tabular}

\section{Resultados}

Tras proceder con el algoritmo de generación y descarte de configuraciones, se ha reducido todo el conjunto inicial de combinaciones matriciales a únicamente cinco arquitecturas válidas (Figura 2). La primera de las configuraciones obtenidas es la conexión en cascada anteriormente comentada. Sin embargo, algunas de las otras configuraciones presentan cualidades interesantes como la posibilidad de la compensación de la tensión de la batería principal con un pack en serie (Figura 2, e), la posibilidad de tener baterías intercambiables mediante caminos conductivos sin conversión y por tanto, sin pérdidas (Figura 2, c), o el control completo de los flujos de energía en cada batería (Figura 2, b).

\section{Conclusiones y trabajo futuro}

Este estudio muestra un método de síntesis de arquitecturas de bloques basado en un conjunto de reglas aplicadas a la hibridación de sistemas de almacenamiento de energía. El método desarrollado permite el cálculo de todas las arquitecturas posibles, y mediante él se han hallado configuraciones no estudiadas en el estado del arte, algunas de las cuales presentan interesantes cualidades.

Además de los resultados aquí recogidos, este algoritmo se ha extendido con éxito a otras casuísticas, con un mayor número de elementos. El siguiente paso en este estudio es abordar el diseño topológico de los convertidores en las arquitecturas más interesantes de entre las sintetizadas.

\section{REFERENCIAS}

[1] KUTKUT, N.H. and DIVAN, D.M. Dynamic equalization techniques for series battery stacks. In : Proceedings of Intelec'96 - International Telecommunications Energy Conference. IEEE, 1996. p. 514-521. ISBN 0-7803-3507-4.

[2] LUKIC, S.M., JIAN CAO, BANSAL, R.C., RODRIGUEZ, F. and EMADI, A. Energy Storage Systems for Automotive Applications. IEEE Transactions on Industrial Electronics. June 2008. Vol. 55, no. 6, p. 2258-2267. DOI 10.1109/TIE.2008.918390.

[3] KIM, Younghyun, KOH, Jason, XIE, Qing, WANG, Yanzhi, CHANG, Naehyuck and PEDRAM, Massoud. A scalable and flexible hybrid energy storage system design and implementation. Journal of Power Sources. 2014. Vol. 255, p. 410-422.

DOI 10.1016/j.jpowsour.2013.12.102.
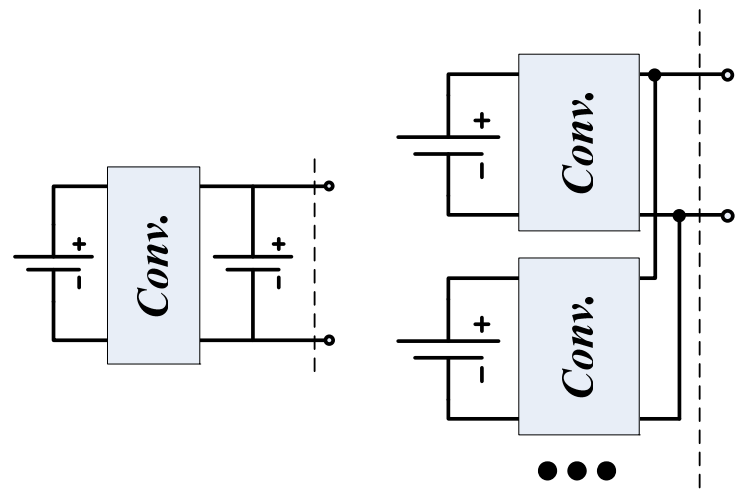

(a)

(b)

Figura 1. Arquitecturas habitualmente utilizadas

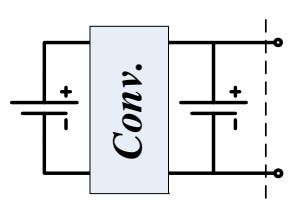

(a)

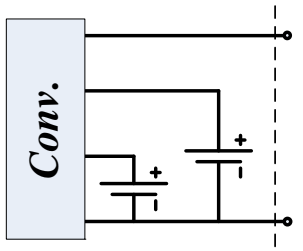

(b)

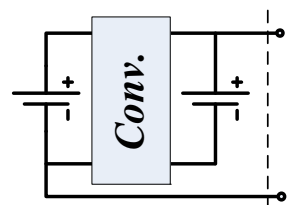

(c)

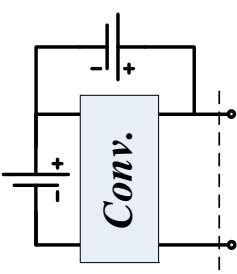

(d)

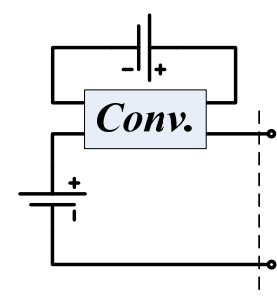

(e)

Figura 2. Configuraciones de arquitectura obtenidas con el algoritmo 